\title{
Recent BaBar results in light hadron spectroscopy
}

\author{
Evgeny Kozyrev ${ }^{1,2, *}$, Evgeny Solodov ${ }^{1,2}$, Vladimir Druzhinin ${ }^{1,2}$, and on behalf of BaBar \\ Collaboration \\ ${ }^{1}$ Budker Institute of Nuclear Physics SB RAS, Novosibirsk 630090 \\ ${ }^{2}$ Novosibirsk State University, Novosibirsk 630090
}

\begin{abstract}
We report the results on the channels $e^{+} e^{-} \rightarrow \pi^{+} \pi^{-} \pi^{0} \pi^{0} \pi^{0}(\eta)$, $e^{+} e^{-} \rightarrow \pi^{+} \pi^{-} \eta$. Also we present the study of the two-photon process $e^{+} e^{-} \rightarrow$ $e^{+} e^{-} \eta^{\prime}$ in the double-tag mode. The results for the form factor are compared with the predictions based on pQCD and VMD. The results are obtained by using the full data set of about $470 \mathrm{fb}^{-1}$ collected by the BABAR experiment at the PEP-II $e^{+} e^{-}$collider at a center-of-mass energy of about $10.6 \mathrm{GeV}$.
\end{abstract}

\section{Introduction}

Uncertainties on vacuum polarization and on the data of two photon interaction are a limiting factor in precise comparisons of data with the expectations of the Standard Model, such as the value of the muon magnetic moment anomaly $a_{\mu}$. In this article we report the results on the measurements of the channels $e^{+} e^{-} \rightarrow \pi^{+} \pi^{-} \pi^{0} \pi^{0} \pi^{0}(\eta)$ [1], $e^{+} e^{-} \rightarrow \pi^{+} \pi^{-} \eta$ [2] and $e^{+} e^{-} \rightarrow e^{+} e^{-} \eta^{\prime}[3]$.

\section{$2 e^{+} e^{-} \rightarrow \pi^{+} \pi^{-} \pi^{0} \pi^{0} \pi^{0}$}

The previous measurements of the cross section of $e^{+} e^{-} \rightarrow \pi^{+} \pi^{-} \pi^{0} \pi^{0} \pi^{0}$ were performed with very limited precision with detectors (M3N [4], MEA [5]). We simulate this channel assuming production through the $\omega(782) \pi^{0} \pi^{0}$ and $\eta \rho$ intermediate channels, with decay of the $\omega(782)$ to three pions and decay of the $\eta$ to all its measured decay modes. The reconstruction efficiency is about $4 \%$, roughly independent of mass. Measured cross section of the process is shown in Fig. 1. The cross section exhibits a structure around $1.7 \mathrm{GeV}$ with a peak value of about $2.5 \mathrm{nb}$, followed by a monotonic decrease toward higher energies. Also we measured for the fist time the values $B\left(J / \psi \rightarrow \pi^{+} \pi^{-} \pi^{0} \pi^{0} \pi^{0}\right)=(27.0 \pm 0.7 \pm 2.7) \times 10^{-3}$ and $B(\psi(2 S) \rightarrow$ $\left.\pi^{+} \pi^{-} \pi^{0} \pi^{0} \pi^{0}\right)=(5.2 \pm 0.8 \pm 0.5) \times 10^{-3}$. Figure 2 shows the number of $\eta \pi^{+} \pi^{-}$(triangles), $\omega \pi^{0} \pi^{0}$ (upside-down triangles), and $\rho^{ \pm} \pi^{\mp} \pi^{0} \pi^{0}$ (squares) intermediate state events. Besides events with single $\rho$ meson production there are more than 50\% events with two $\rho$ mesons $\rho^{+} \rho^{-} \pi^{0}$. Amplitudes with $\rho$ meson(s) are totally dominated above $2 \mathrm{GeV}$.

\footnotetext{
*e-mail: e.a.kozyrev@inp.nsk.su
} 


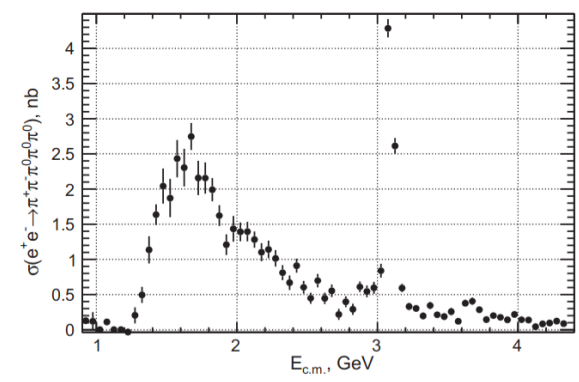

Figure 1. The measured $e^{+} e^{-} \rightarrow \pi^{+} \pi^{-} \pi^{0} \pi^{0} \pi^{0}$ cross section. The uncertainties are statistical only. The low energy region $\left(E_{c . m .}<2 \mathrm{GeV}\right)$ is dominated by $\eta \pi^{+} \pi^{-}$and $\omega \pi^{0} \pi^{0}$ amplitudes, while $\left(\rho^{ \pm} \pi^{\mp} \pi^{0} \pi^{0}\right)$ dominates at higher energies.

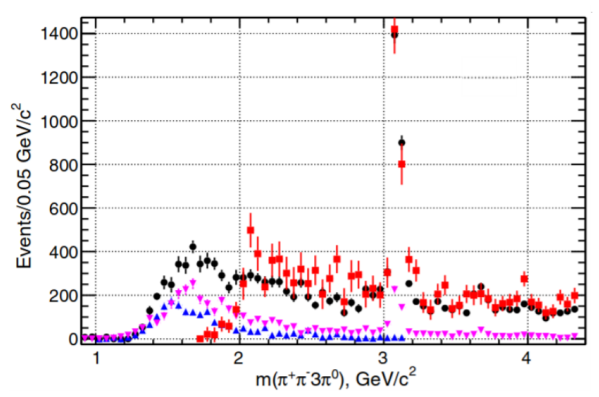

Figure 2. Number of events in bins of $\mathrm{E}_{c . m}$. from the $\eta \pi^{+} \pi^{-}$(triangles), $\omega \pi^{0} \pi^{0}$ (upsidedown triangles), and $\rho^{ \pm} \pi^{\mp} \pi^{0} \pi^{0}$ (squares) intermediate states. The circles show the total event numbers of signal events.

\section{$3 e^{+} e^{-} \rightarrow \pi^{+} \pi^{-} \pi^{0} \pi^{0} \eta$}

The study of this process was performed in the same way as described in Sec. 2. This cross section was measured for the first time and is shown in Fig. 3. The process is dominated by the intermediate states $\omega \pi^{0} \eta, \phi \pi^{0} \eta, \rho(770)^{ \pm} \pi^{\mp} \pi^{0} \eta$ and $\rho(770)^{+} \rho(770)^{-} \eta$. The signal from $a_{0}(980) \rho \pi$ is seen. The data also allowed to measure for the first time the branching fraction $B\left(J / \psi \rightarrow \pi^{+} \pi^{-} \pi^{0} \pi^{0} \eta\right)=(2.30 \pm 0.33 \pm 0.35) \times 10^{-3}$ and set the limit $B\left(\psi(2 S) \rightarrow \pi^{+} \pi^{-} \pi^{0} \pi^{0} \eta\right)<$ $0.35 \times 10^{-3}$ at $90 \%$ C.L.

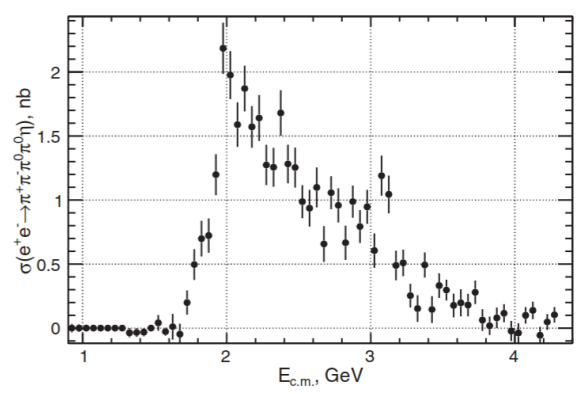

Figure 3. The measured $e^{+} e^{-} \rightarrow \pi^{+} \pi^{-} \pi^{0} \pi^{0} \eta$ cross section. The uncertainties are statistical only.

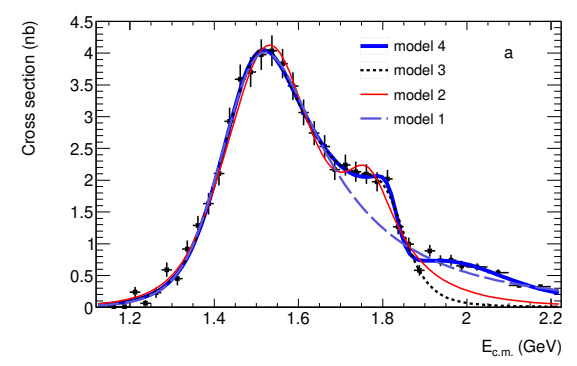

Figure 4. The measured $e^{+} e^{-} \rightarrow \pi^{+} \pi^{-} \eta$ cross section fitted with the four models described in the text.

\section{$4 e^{+} e^{-} \rightarrow \pi^{+} \pi^{-} \eta$}

This process was studied with BaBar detector in three main decay modes: $\eta \rightarrow 3 \pi^{0}[1]$, $\eta \rightarrow 2 \gamma[2]$ and $\eta \rightarrow \pi^{+} \pi^{-} \pi^{0}$ [7].

The cross section of $e^{+} e^{-} \rightarrow \pi^{+} \pi^{-} \eta(\rightarrow 2 \gamma)$ was approximated by the VMD as shown in Fig. 4. The models with one, two, and three excited states are tested. In Model 1, the cross section data are fitted in the energy range $E_{c . m} .=1.2-1.70 \mathrm{GeV}$ with two resonances, $\rho(770)$ and $\rho(1450)$. The model with $\phi_{\rho(1450)}=0$ fails to describe the data. The fit result 
with $\phi_{\rho(1450)}=\pi$ is shown in Fig. 4 by the long-dashed curve. It is seen that Model 1 cannot reproduce the structure in the cross section near $1.8 \mathrm{GeV}$.

In Models 2 and 3 we include an additional contribution from the $\rho(1700)$ resonance with phases $\phi_{\rho(1700)}=\pi$ and 0 , respectively. The fits are done in the range $E_{c . m .}=1.2-1.90 \mathrm{GeV}$. Both models describe the data below $1.90 \mathrm{GeV}$ reasonably well. Model 3 has better $\chi^{2}$ $\left(P\left(\chi^{2}\right)=0.58\right.$ instead of 0.03 for Model 2). Above $1.90 \mathrm{GeV}$ the fit curves for both the models lie below the data.

Model 4 is Model 3 with a fourth resonance $\rho^{\prime \prime \prime}$ added. The phase $\phi_{\rho^{\prime \prime \prime}}$ is set to zero. The fitted energy range is extended up to $2.2 \mathrm{GeV}$. The fit result is shown in Fig. 4. The fitted resonance mass $m_{\rho^{\prime \prime \prime}}=2.01 \pm 0.04 \mathrm{GeV}$ is between the masses of the $\rho(1900)$ and $\rho(2150)$ states listed in the PDG table [10]. The fitted value $g_{\rho}=1.7 \pm 0.3 \mathrm{GeV}^{-1}$ agrees with the VMD estimation of $1.57 \pm 0.07 \mathrm{GeV}^{-1}$ from the partial width $\rho(770) \rightarrow \eta \gamma$. It is seen that the model successfully describes the cross section data up to $2.2 \mathrm{GeV}$. Above $E_{c . m}$. $=2.3 \mathrm{GeV}$ Model 4 lies below the data, which could be explained by another resonance. Alternatively, the change of the cross section slope near $1.9 \mathrm{GeV}$ may be interpreted without inclusion of a fourth resonance, as a threshold effect due to the opening of the nucleon-antinucleon production channel.

From the fit in Model 3 we obtain:

$$
\begin{gathered}
\Gamma\left(\rho(1450) \rightarrow e^{+} e^{-}\right) B\left(\rho(1450) \rightarrow \eta \pi^{+} \pi^{-}\right)=\left(210 \pm 24_{\text {stat }} \pm 10_{\text {syst }}\right) \mathrm{eV} \\
\Gamma\left(\rho(1700) \rightarrow e^{+} e^{-}\right) B\left(\rho(1700) \rightarrow \eta \pi^{+} \pi^{-}\right)=\left(84 \pm 26_{\text {stat }} \pm 4_{\text {syst }}\right) \mathrm{eV}
\end{gathered}
$$

The model uncertainties of these parameters estimated from the difference of fit results for Model 2, 3, and 4, are large, 20\% for $\rho(1450)$ and $80 \%$ for $\rho(1700)$.

The CVC hypothesis and isospin symmetry allow the prediction of the $\pi^{-} \pi^{0} \eta$ mass spectrum and the branching fraction for the $\tau^{-} \rightarrow \pi^{-} \pi^{0} \eta \nu_{\tau}$ decay from data for the $e^{+} e^{-} \rightarrow \pi^{+} \pi^{-} \eta$ cross section [6]. The branching fraction can be calculated as:

$$
\frac{B\left(\tau^{-} \rightarrow \pi^{-} \pi^{0} \eta v_{\tau}\right)}{B\left(\tau^{-} \rightarrow e^{-} \overline{v_{e}} \nu_{\tau}\right)}=\int_{\left(2 m_{\pi}+m_{\eta}\right)^{2}}^{m_{\tau}^{2}} d q^{2} \sigma_{e^{+} e^{-} \rightarrow \pi^{+} \pi^{-} \eta}^{I=1}\left(q^{2}\right) \frac{3\left|V_{u d}\right|^{2} S_{\mathrm{EW}}}{2 \pi \alpha^{2}} \frac{q^{2}}{m_{\tau}^{2}}\left(1-\frac{q^{2}}{m_{\tau}^{2}}\right)^{2}\left(1+2 \frac{q^{2}}{m_{\tau}^{2}}\right)
$$

where $q^{2}$ is the squared 4-momentum of the $\pi^{ \pm} \pi^{0} \eta$ system, $\left|V_{u d}\right|$ is the Cabibbo-KobayashiMaskawa matrix element, and $S_{\mathrm{EW}}=1.0194$ is a factor taking into account electroweak radiative corrections, and $B\left(\tau^{-} \rightarrow e^{-} \overline{v_{e}} v_{\tau}\right)=17.83 \pm 0.04 \%$ [10].

We integrate Eq.(2) using the fit function for the cross section of Model 4 and obtain

$$
\begin{gathered}
B\left(\tau^{-} \rightarrow \pi^{-} \pi^{0} \eta v_{\tau}\right)=\left(0.1616 \pm 0.0026_{\text {stat }} \pm 0.0080_{\text {syst }} \pm 0.0011_{\text {model }}\right) \% \\
=(0.162 \pm 0.009) \%,
\end{gathered}
$$

where the first error is statistical, the second is systematic, and the third is model uncertainty. The latter is estimated from the difference between the branching fraction values obtained with the cross section parametrization in Model 2 and Model 3 discussed in the previous section.

The calculation based on the previous BaBar measurement of the $\pi^{+} \pi^{-} \eta \rightarrow \pi^{+} \pi^{-} \pi^{+} \pi^{-} \pi^{0}$ final state [1] gives $B\left(\tau^{ \pm} \rightarrow \pi^{ \pm} \pi^{0} \eta v_{\tau}\right)=\left(0.1695 \pm 0.0085_{\text {stat }} \pm 0.0136_{\text {syst }}\right) \%$, compatible with the result (3). The systematic uncertanties on the luminosity, radiative corrections, photon and track efficiencies are the same for the new and previous BaBar measurements. Combining the two BaBar values we obtain

$$
B\left(\tau^{-} \rightarrow \pi^{-} \pi^{0} \eta v_{\tau}\right)=(0.163 \pm 0.008) \%
$$




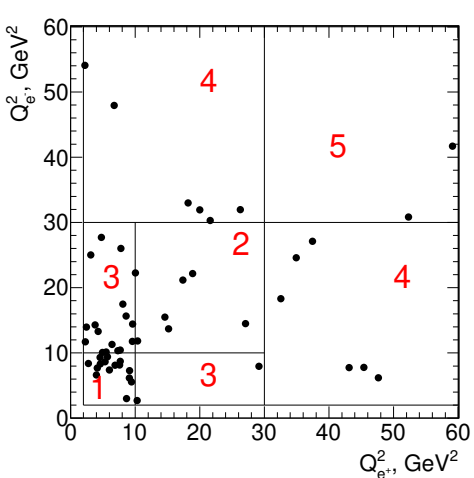

Figure 5. The $Q_{e^{-}}^{2}$ versus $Q_{e^{+}}^{2}$ distribution for data events. The lines and numbers indicate the five regions used for the study of the dynamics of TFF a function of $Q_{e^{-}}^{2}$ and $Q_{e^{+}}^{2}$.

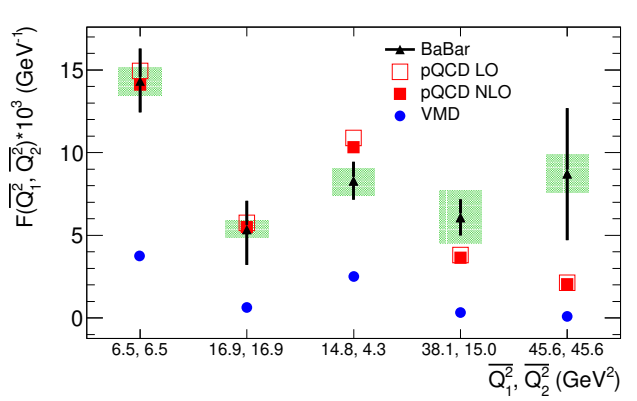

Figure 6. Comparison of the measured $\gamma^{\star} \gamma^{\star} \rightarrow$ $\eta^{\prime}$ transition form factor (triangles, with error bars representing the statistical uncertainties) with the LO (open squares) and NLO (filled squares) pQCD predictions and the VDM predictions (circles).

which is in good agreement with, but more precise than, the estimate based on the SND $e^{+} e^{-} \rightarrow \pi^{+} \pi^{-} \eta$ measurement $(0.156 \pm 0.011) \%$ [11].

The PDG value of this branching fraction is $B\left(\tau^{-} \rightarrow \pi^{-} \pi^{0} \eta v_{\tau}\right)_{\exp }=(0.139 \pm 0.010) \%$ [10]. The difference between the experimental result and our CVC-based calculation is $1.8 \sigma$. The difference, about $15 \%$ of the branching fraction, is too large to be explained by isospinbreaking corrections.

\section{$5 e^{+} e^{-} \rightarrow e^{+} e^{-} \eta^{\prime}$}

In this section we report on the measurement of the $\gamma^{\star} \gamma^{\star} \rightarrow \eta^{\prime}$ transition form factor (TFF) by using the two-photon-fusion reaction $e^{+} e^{-} \rightarrow e^{+} e^{-} \eta^{\prime}$. The TFF is defined via the amplitude for the $\gamma^{\star} \gamma^{\star} \rightarrow \eta^{\prime}$ transition

$$
T=-i 4 \pi \alpha \epsilon_{\mu \nu \beta \gamma} \varepsilon_{1}^{\mu} \varepsilon_{2}^{v} q_{1}^{\beta} q_{2}^{\gamma} F_{\eta^{\prime}}\left(Q_{1}^{2}, Q_{2}^{2}\right),
$$

where $\alpha$ is the fine structure constant, $\epsilon_{\mu v \alpha \beta}$ is the totally antisymmetric Levi-Civita tensor, $\varepsilon_{1,2}$ and $q_{1,2}$ are the polarization vectors and four-momenta, respectively, of the space-like photons, $Q_{1,2}^{2}=-q_{1,2}^{2}$, and $F_{\eta^{\prime}}\left(Q_{1}^{2}, Q_{2}^{2}\right)$ is the transition form factor.

We measure the differential cross section of the process $e^{+} e^{-} \rightarrow e^{+} e^{-} \eta^{\prime}$ in the double-tag mode, in which both scattered fermions are detected (tagged). The tagged electrons emit highly off-shell photons with momentum transfers $q_{e^{ \pm}}^{2}=-Q_{e^{ \pm}}^{2}=\left(p_{e^{ \pm}}-p_{e^{ \pm}}^{\prime}\right)^{2}$, where $p_{e^{ \pm}}$and $p_{e^{ \pm}}^{\prime}$ are the four-momenta, respectively, of the initial- and final-state electrons. We measure for the first time $F_{\eta^{\prime}}\left(Q_{1}^{2}, Q_{2}^{2}\right)$ in the kinematic region with two highly off-shell photons $2<$ $Q_{1}^{2}, Q_{2}^{2}<60 \mathrm{GeV}^{2}$. This measurement of the TFF at large $Q_{1}^{2}$ and $Q_{2}^{2}$ allows the predictions of models inspired by perturbative QCD (pQCD) to be distinguished from those of the vector dominance model (VDM) [8, 9]. In the case of only one off-shell photon, both classes of models predict the same asymptotic dependence $F_{P}\left(Q^{2}, 0\right) \sim 1 / Q^{2}$ as $Q^{2} \rightarrow \infty$, while for two off-shell photons the asymptotic predictions are quite different, $F\left(Q_{1}^{2}, Q_{2}^{2}\right) \sim 1 /\left(Q_{1}^{2}+Q_{2}^{2}\right)$ for pQCD, and $F\left(Q_{1}^{2}, Q_{2}^{2}\right) \sim 1 /\left(Q_{1}^{2} Q_{2}^{2}\right)$ for the VDM model. 
The decay chain $\eta^{\prime} \rightarrow \pi^{+} \pi^{-} \eta \rightarrow \pi^{+} \pi^{-} 2 \gamma$ is used to reconstruct the $\eta^{\prime}$ meson candidate. Data events that pass all selection criteria are divided into five $\left(Q_{e^{-}}^{2}, Q_{e^{+}}^{2}\right)$ regions, as illustrated on Fig. 5 for events with $0.945<M_{\pi^{+} \pi^{-} \eta}<0.972 \mathrm{GeV} / \mathrm{c}^{2}$. Because of the symmetry of the process under the exchange of the $e^{-}$with the $e^{+}$, regions 3 and 4 each include two disjunct regions, mirror symmetric with respect to the diagonal. The number of signal events in each $\left(Q_{e^{-}}^{2}, Q_{e^{+}}^{2}\right)$ region is obtained from a fit to the $\pi^{+} \pi^{-} \eta$ invariant mass spectrum with a sum of signal and background distributions. The total number of signal events is $46.2_{-7.0}^{+8.3}$. The total systematic uncertainty related to the description of the background and signal is $3.7 \%$ and the total systematic uncertainty of the detection efficiency is $11 \%$. The obtained values of the transition form factor are published in [3] and are represented in Fig. 6 by the triangles. The error bars attached to the triangles indicate the statistical uncertainties. The quadratic sum of the systematic and model uncertainties is shown by the shaded rectangles. The open and filled squares in Fig. 6 correspond to the LO and NLO pQCD predictions, respectively. The NLO correction is relatively small. The measured TFF is, in general, consistent with the QCD prediction. The circles in Fig. 6 represent the predictions of the VDM model, which exhibits a clear disagreement with the data.

\section{Summary}

Recent results on the study on the channels $e^{+} e^{-} \rightarrow \pi^{+} \pi^{-} \pi^{0} \pi^{0} \pi^{0}(\eta), e^{+} e^{-} \rightarrow \pi^{+} \pi^{-} \eta$ and $e^{+} e^{-} \rightarrow e^{+} e^{-} \eta^{\prime}$ obtained with BaBar detector are presented in the paper. For this research we have received support from the Russian Foundation for Basic Research (grant No. 18-3201020).

\section{References}

[1] J. P. Lees et al. (BaBar Collaboration), Phys. Rev. D 98, 112015 (2018).

[2] J. P. Lees et al. (BaBar Collaboration), Phys. Rev. D 97 ,052007 (2018).

[3] J. P. Lees et al. (BaBar Collaboration), Phys. Rev. D 98, 112002 (2018).

[4] G. Cosmeet et al., Nucl. Phys. B 152, 215 (1979).

[5] B. Espositoet et al., Lett. Nuovo Cimento 25, 5 (1979).

[6] Y. S. Tsai, Phys. Rev. D 4, 2821 (1971).

[7] B. Aubert et al. (BABAR Collaboration), Phys. Rev. D 76, 092005 (2007).

[8] B.-1. Young, Phys. Rev. 161, 1620 (1967).

[9] L. G. Landsberg, Phys. Rep. 128, 301 (1985).

[10] C. Patrignani et al. (Particle Data Group), Chin. Phys. C 40, 100001 (2016).

[11] V. M. Aulchenko et al. (SND Collaboration), Phys. Rev. D 91, 052013 (2015). 